\title{
NON-LINEAR OSCILLATIONS OF PENDULUM WITH VERTICALLY MOVING MULTIFREQUENCY SUPPORT POINT
}

\author{
TRAN KIM CHI \\ Institute of Applied Mechanics
}

In this paper the problem of nonlinear oscillations of the pendulum with moving two frequency support point [1] is extended to the case of vertical multifrequency motion of the support point by means of the asymptotic method of nonlinear oscillations [2]. The stability conditions of the equilibrium positions of the pendulum are delivered.

\section{§1. EQUATION OF MOTION}

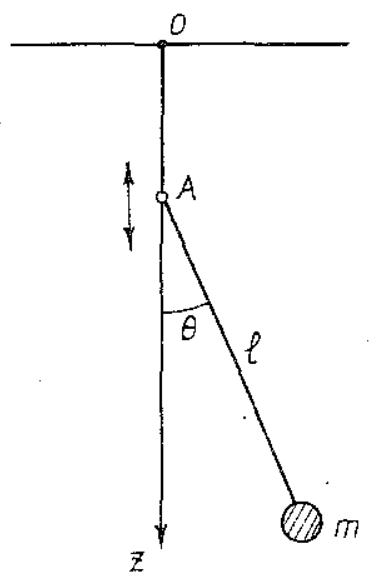

Let us consider a mathematical pendulum of length $\ell$, mass $m$, whose support point A nioves vertically by the law:

$$
O A=Z_{A}=a_{1} \sin \left(\omega_{1} t+\alpha_{1}\right)+a_{2} \sin \left(\omega_{2} t+\alpha_{2}\right)+\cdots+a_{N} \sin \left(\omega_{N} t+\alpha_{N}\right),
$$

where $a_{i}, \omega_{i}, \alpha_{i}$ are constants.

The equation of motion of the pendulum is:

$$
m \ell^{2} \ddot{\theta}+h \dot{\theta}+m \ell\left(g-\ddot{Z}_{A}\right) \sin \theta=0,
$$

where overdots denote the derivatives relatively to time.

Dividing equation (1.1) by $m \ell^{2} \omega^{2}$ and introducing the notations:

$$
\omega_{0}^{2}=\frac{g}{\ell}, \quad H=\frac{h}{m \ell^{2}}, \quad \tau=\omega t, \quad t=\frac{d}{d \tau}, \quad e_{i}=\frac{a_{i}}{a}, \quad \nu_{i}=\frac{\omega_{i}}{\omega},
$$


where $\omega$ is a positive number which characterises the average value of $\omega_{1}, \omega_{2}, \ldots, \omega_{N}$ and $a$ is average value of $a_{1}, a_{2}, \ldots, a_{N}$, and $h$ is coefficient of friction, we can write (1.1) in the form:

$\theta^{\prime \prime}+\frac{H}{\omega} \theta^{\prime}+\left\{\frac{\omega_{0}^{2}}{\omega^{2}}+\frac{a}{\ell}\left[e_{1} \nu_{1}^{2} \sin \left(\nu_{1} \tau+\alpha_{1}\right)+e_{2} \nu_{2}^{2} \sin \left(\nu_{2} \tau+\alpha_{2}\right)+\cdots+e_{N} \nu_{N}^{2} \sin \left(\nu_{N} \tau+\alpha_{N}\right)\right]\right\} \sin \theta=0$

It is suppopsed that $a / \ell, \omega_{0} / \omega$ and $H / \omega_{0}$ are small quantities of $\varepsilon$-degree, where $\varepsilon$ is a small positive parameter, so that

$$
\varepsilon=\frac{a}{\ell}, \quad \frac{\omega_{0}}{\omega}=k \varepsilon, \quad \frac{H}{\omega}=2 \lambda \varepsilon^{2}
$$

We have

$\theta^{\prime \prime}+2 \lambda \varepsilon^{2} \theta^{\prime}+\left\{\varepsilon^{2} k^{2}+\varepsilon\left[e_{1} \nu_{1}^{2} \sin \left(\nu_{1} \tau+\alpha_{1}\right)+e_{2} \nu_{2}^{2} \sin \left(\nu_{2} \tau+\alpha_{2}\right)+\cdots+e_{N} \nu_{N}^{2} \sin \left(\nu_{N} \tau+\alpha_{N}\right)\right]\right\} \sin \theta=0$

Using the new variables $\varphi$ and $\Omega$ instead of $\theta$ and $\theta^{\prime}$ by formulae $[2]$

$$
\begin{aligned}
\theta & =\varphi+\varepsilon\left[e_{1} \sin \left(\nu_{1} \tau+\alpha_{1}\right)+e_{2} \sin \left(\nu_{2} \tau+\alpha_{2}\right)+\cdots+e_{N} \sin \left(\nu_{N} \tau+\alpha_{N}\right)\right] \sin \varphi \\
\theta^{\prime} & =\varepsilon \Omega+\varepsilon\left[e_{1} \nu_{1} \cos \left(\nu_{1} \tau+\alpha_{1}\right)+e_{2} \nu_{2} \cos \left(\nu_{2} \tau+\alpha_{2}\right)+\cdots+e_{N} \nu_{N} \cos \left(\nu_{N} \tau+\alpha_{N}\right)\right] \sin \varphi
\end{aligned}
$$

one can transform the equation (1.5) into a system of two equations of the first order:

$$
\begin{aligned}
\varphi^{\prime}= & \varepsilon \Omega-\varepsilon^{2} \Omega I_{1} \cos \varphi, \\
\Omega^{\prime}= & -\varepsilon\left(\Omega I_{2} \cos \varphi+k^{2} \sin \varphi+I_{1} I_{3} \sin \varphi \cos \varphi\right)+\varepsilon^{2}\left(\Omega I_{1} I_{2} \cos ^{2} \varphi-\right. \\
& \left.-2 \lambda \Omega-2 \lambda I_{2} \sin \varphi-k^{2} I_{1} \sin \varphi \cos \varphi+\frac{1}{2} I_{1}^{2} I_{3} \sin ^{3} \varphi\right),
\end{aligned}
$$

where the terms with degree of smallness higher than two are neglected,

$$
I_{1}=\sum_{i=1}^{N} e_{i} \sin \left(\nu_{i} \tau+\alpha_{i}\right) ; \quad I_{2}=\sum_{i=1}^{N} e_{i} \nu_{i} \cos \left(\nu_{i} \tau+\alpha_{i}\right) ; \quad I_{3}=\sum_{i=1}^{N} e_{i} \nu_{i}^{2} \sin \left(\nu_{i} \tau+\alpha_{i}\right)
$$

\section{§2. APPROXIMATE SOLUTION IN THE NON-RESONANCE CASE}

Let us consider the non-resonance case when the frequencies $\nu_{1}, \nu_{2}, \ldots, \nu_{N}$ are linearly independent, so that between them there is no relation of the form

$$
n_{1} \nu_{1}+n_{2} \nu_{2}+\cdots+n_{N} \dot{\nu}_{N}=0
$$

where $n_{i}$ are integers, $n_{1}^{2}+n_{2}^{2}+\cdots+n_{N}^{2} \neq 0$. Since the variables $\varphi$ and $\Omega$ are slowly varying in $\tau$, in the first approximation one can replace the right hand sides of $(1.7)$ by their average values. We have in the first approximation

$$
\varphi=\varphi_{1}, \quad \Omega=\Omega_{1},
$$

where $\varphi_{1}$ and $\Omega_{1}$ satisfy the averaged equations: 


$$
\begin{aligned}
& \varphi_{1}^{\prime}=\dot{\varepsilon} \Omega_{1}, \\
& \Omega_{1}^{\prime}=-\varepsilon\left[k^{2} \sin \varphi_{1}+\frac{1}{4}\left(e_{1}^{2} \nu_{1}^{2}+e_{2}^{2} \nu_{2}^{2}+\cdots+e_{N}^{2} \nu_{N}^{2}\right) \sin 2 \varphi_{1}\right] .
\end{aligned}
$$

The stationary solutions of (2.3) are

$$
\text { 1) } \Omega_{1}=0, \quad \varphi_{1}=0,
$$

(downward vertical position of equilibrium : $\theta=\dot{\theta}=0$ )

$$
\text { 2) } \Omega_{1}=0, \quad \varphi_{1}=\pi \text {, }
$$

(upward vertical position of equilibrium : $\theta=\pi, \dot{\theta}=0$ )

$$
\text { 3) } \Omega_{1}=0, \varphi_{1}=\varphi_{0} \text {, }
$$

determined by the relation

$$
k^{2}+\frac{1}{2}\left(e_{1}^{2} \nu_{1}^{2}+e_{2}^{2} \nu_{2}^{2}+\cdots+e_{N}^{2} \nu_{N}^{2}\right) \cos \varphi_{0}=0 .
$$

This solution exists if

$$
k^{2} \leq \frac{1}{2}\left(e_{1}^{2} \nu_{1}^{2}+e_{2}^{2} \nu_{2}^{2}+\cdots+e_{N}^{2} \nu_{N}^{2}\right) .
$$

To study the stability of the stationary solutions we use the variational equations. Introducing the variations:

$$
\delta \Omega=\Omega_{1}, \quad \delta \varphi=\varphi_{1}-\varphi^{*},
$$

where $\varphi^{*}=0, \varphi^{*}=\pi$ and $\varphi^{*}=\varphi_{0}$ for the cases $(2.4),(2.5)$ and (2.6) respectively, we have

$$
\begin{aligned}
& \frac{d}{d \tau} \delta \varphi=\varepsilon \delta \Omega, \\
& \frac{d}{d \tau} \delta \Omega=-\varepsilon\left[k^{2} \cos \varphi^{*}+\frac{1}{2}\left(e_{1}^{2} \nu_{1}^{2}+e_{2}^{2} \nu_{2}^{2}+\cdots+e_{N}^{2} \nu_{N}^{2}\right) \cos 2 \varphi^{*}\right] \delta \varphi, \\
& \frac{d^{2}}{d \tau^{2}} \delta \varphi+\varepsilon^{2}\left[k^{2} \cos \varphi^{*}+\frac{1}{2}\left(e_{1}^{2} \nu_{1}^{2}+e_{2}^{2} \nu_{2}^{2}+\cdots+e_{N}^{2} \nu_{N}^{2}\right) \cos 2 \varphi^{*}\right] \delta \varphi=0 .
\end{aligned}
$$

The necessary stability condition of the stationary solution is:

$$
k^{2} \cos \varphi^{*}+\frac{1}{2}\left(e_{1}^{2} \nu_{1}^{2}+e_{2}^{2} \nu_{2}^{2}+\cdots+e_{N}^{2} \nu_{N}^{2}\right) \cos 2 \varphi^{*}>0 .
$$

Namely,

1. For $\varphi^{*}=0$ the condition (2.11) is always satisfied.

2. For $\varphi^{*}=\pi$ the necessary stability condition becomes:

$$
k^{2}<\frac{1}{2}\left(e_{1}^{2} \nu_{1}^{2}+e_{2}^{2} \nu_{2}^{2}+\cdots+e_{N}^{2} \nu_{N}^{2}\right)
$$

or taking into account (1.2), (1.4):

$$
\omega_{0}^{2}<\frac{1}{2}\left[\left(\frac{a_{1}}{\ell}\right)^{2} \omega_{1}^{2}+\cdots+\left(\frac{a_{N}}{\ell}\right)^{2} \omega_{N}^{2}\right] .
$$

3. For $\varphi^{*}=\varphi_{0}$ the necessary stability condition is 


$$
\omega_{0}^{2}>\frac{1}{2}\left[\left(\frac{a_{1}}{\ell}\right)^{2} \omega_{1}^{2}+\cdots+\left(\frac{a_{N}}{\ell}\right)^{2} \omega_{N}^{2}\right]
$$

which is in contrary with (2.8).

Since the characteristic equation of $(2.10)$ has zero real parts, to solve completely the stability problem of stationary solution it is necessary to consider the higher approximation.

The refinement of the first approximation of solutions of (1.7) is:

$$
\begin{aligned}
\varphi= & \varphi_{1}, \\
\Omega= & \Omega_{1}-\varepsilon\left\{\Omega_{1} \cos \varphi_{1} \sum_{j=1}^{N} e_{j} \sin \xi_{j}-\frac{1}{8} \sin 2 \varphi_{1} \sum_{j=1}^{N} \nu_{j} e_{j}^{2} \sin 2 \xi_{j}+\right. \\
& \left.+\frac{1}{4} \sin 2 \varphi_{1} \sum_{\substack{i=1 \\
i \neq j}}^{N} \sum_{j=1}^{N} e_{i} e_{j} \nu_{j}^{2}\left[\frac{1}{\nu_{i}-\nu_{j}} \sin \left(\xi_{i}-\xi_{j}\right)-\frac{1}{\nu_{i}+\nu_{j}} \sin \left(\xi_{i}+\xi_{j}\right)\right]\right\},
\end{aligned}
$$

where $\xi_{i}=\nu_{i} \tau+\alpha_{i}$.

Substituting (2.15) into (1.7) and averaging their right hand sides we obtain the equations of the second approximation

$$
\begin{aligned}
& \varphi_{1}^{\prime}=\varepsilon \Omega_{1}, \\
& \Omega_{1}^{\prime}=-\varepsilon\left[k^{2} \sin \varphi_{1}+\frac{1}{4}\left(e_{1}^{2} \nu_{1}^{2}+\cdots+e_{N}^{2} \nu_{N}^{2}\right) \sin 2 \varphi_{1}\right]-2 \varepsilon^{2} \lambda \Omega_{1},
\end{aligned}
$$

The stationary solutions of these equations are as before $(2.4),(2.5)$ and $(2.6)$, but the variational equation for them is of the form:

$$
\frac{d^{2}}{d \tau^{2}} \delta \varphi_{1}+2 \varepsilon^{2} \lambda \frac{d}{d \tau} \delta \varphi_{1}+\varepsilon^{2}\left[k^{2} \cos \varphi^{*}+\frac{1}{2}\left(e_{1}^{2} \nu_{1}^{2}+\cdots+e_{N}^{2} \nu_{N}^{2}\right) \cos 2 \varphi^{*}\right] \delta \varphi_{1}=0
$$

which is different from $(2.9)$ by the appearance of the term $2 \varepsilon^{2} \lambda \frac{d}{d \tau} \delta \varphi_{1}\left(\varphi^{*}=0, \pi, \varphi_{0}\right)$. The necessary and sufficient stability condition for the stationary solutions $(2.4),(2.6)$ is:

1. The solution $\Omega_{1}=0, \varphi_{1}=0$ is always stable

2. The solution $\Omega_{1}=0, \varphi_{1}=\pi$ is stable if

$$
k^{2}<\frac{1}{2}\left(e_{1}^{2} \nu_{1}^{2}+\cdots+e_{N}^{2} \nu_{N}^{2}\right)
$$

or if

$$
\omega_{0}^{2}<\frac{1}{2}\left[\left(\frac{a_{1}}{\ell}\right)^{2} \omega_{1}^{2}+\cdots+\left(\frac{a_{N}}{\ell}\right)^{2} \omega_{N}^{2}\right] .
$$

3. The solution $\Omega_{1}=0, \varphi_{1}=\varphi_{0}$ is always unstable.

\section{§3. THE RESONANCE CASE}

It is supposed that between the frequency $\nu_{j}$ and $\nu_{1}$ there exists a relation

$$
\alpha_{j}=2 \nu_{1}, \quad j \neq 1
$$

The other resonance cases can be examined by an analogeous way. Now, the equations of the refinement of the first approximation remain the same as (2.15). Substituting them into (1.7) and averaging on $\tau$ we obtain the following equations of the second approximation 


$$
\begin{aligned}
\varphi_{1}^{\prime}= & \varepsilon \Omega_{1}, \\
\Omega_{1}^{\prime}= & -\varepsilon\left(k^{2} \sin \varphi_{1}+\frac{1}{2} \sin \varphi_{1} \cos \varphi_{1} \sum_{i=1}^{N} e_{i}^{2} \nu_{i}^{2}\right)+\varepsilon^{2}\left\{-2 \lambda \Omega_{1}+\right. \\
& \left.+\frac{e_{1}^{2} e_{j} \nu_{1}\left(\nu_{1}^{2}+\nu_{j}^{2}\right)}{4\left(\nu_{j}-\nu_{1}\right)} \sin \left(\alpha_{j}-2 \alpha_{1}\right) \sin \varphi_{1} \cos ^{2} \varphi_{1}-\frac{1}{8} e_{1}^{2} e_{j}\left(2 \nu_{1}^{2}+\nu_{j}^{2}\right) \sin \left(\alpha_{j}-2 \alpha_{1}\right) \sin ^{3} \varphi_{1}\right\} .
\end{aligned}
$$

The stationary solutions of equations (3.2) are

$$
\begin{aligned}
& \text { 1) } \quad \Omega_{1}=0, \quad \varphi_{1}=0 \text {, } \\
& \text { 2) } \Omega_{1}=0, \quad \varphi_{1}=\pi \text {, } \\
& \text { 3) } \quad \Omega_{1}=0, \quad \varphi_{1}=\varphi_{10}
\end{aligned}
$$

where $\varphi_{10}$ is determined from

$$
\begin{aligned}
k^{2} & +\frac{1}{2} \cos \varphi_{10} \sum_{i=1}^{N} e_{i}^{2} \nu_{i}^{2}+\varepsilon\left\{\frac{e_{1}^{2} e_{j} \nu_{1}\left(\nu_{1}^{2}+\nu_{j}^{2}\right)}{4\left(\nu_{j}-\nu_{1}\right)} \sin \left(\alpha_{j}-2 \alpha_{1}\right) \cos ^{2} \varphi_{10}-\right. \\
& \left.-\frac{1}{8} e_{1}^{2} e_{j}\left(2 \nu_{1}^{2}+\nu_{j}^{2}\right) \sin \left(\alpha_{j}-2 \alpha_{1}\right) \sin ^{2} \varphi_{10}\right\}=0,
\end{aligned}
$$

This solution exists if

$$
k^{2} \leq \frac{1}{2}\left(e_{1}^{2} \nu_{1}^{2}+e_{2}^{2} \nu_{2}^{2}+\cdots+e_{N}^{2} \nu_{N}^{2}\right)
$$

The stability of the stationary solutions (3.3)-(3.5) is studied by using the variational equation of (3.2) which has the following form

$$
\begin{aligned}
& \frac{d^{2}}{d \tau^{2}} \delta \varphi_{1}+2 \lambda \varepsilon^{2} \frac{d}{d \tau} \delta \varphi_{1}+\varepsilon^{2}\left\{k^{2} \cos \varphi_{1}^{*}+\frac{1}{2}\left(e_{1}^{2} \nu_{1}^{2}+\cdots+e_{N}^{2} \nu_{N}^{2}\right) \cos 2 \varphi_{1}^{*}+\right. \\
& \quad+\frac{\varepsilon}{16} \frac{e_{1}^{2} e_{j} \nu_{1}\left(\nu_{1}^{2}+\nu_{j}^{2}\right)}{\left(\nu_{j}-\nu_{1}\right)} \sin \left(\alpha_{j}-2 \alpha_{1}\right)\left[\cos \varphi_{1}^{*}+3 \cos 3 \varphi_{1}^{*}\right]- \\
& \left.\quad-\frac{\varepsilon}{32} e_{1}^{2} e_{j}\left(2 \nu_{1}^{2}+\nu_{j}^{2}\right) \sin \left(\alpha_{j}-2 \alpha_{1}\right)\left[3 \cos \varphi_{1}^{*}-3 \cos 3 \varphi_{1}^{*}\right]\right\} \delta \varphi_{1}=0
\end{aligned}
$$

where $\delta \varphi_{1}=\varphi_{1}-\varphi_{1}^{*}, \quad \varphi_{1}^{*}=0, \pi, \varphi_{10}$.

From (3.7) it follows:

1) For $\varphi_{1}^{*}=0$ and for small $\varepsilon$, the solution $\Omega_{1}=0, \varphi_{1}=0$ (downward vertical position) is stable if:

$$
k^{2}+\frac{1}{2}\left(e_{1}^{2} \nu_{1}^{2}+\cdots+e_{N}^{2} \nu_{N}^{2}\right)>0
$$

This condition is always satisfied.

2) For $\varphi_{1}^{*}=\pi$ and for small $\varepsilon$, the solution $\Omega_{1}=0, \varphi_{1}=\pi$ (upward vertical position) is stable if

$$
k^{2}<\frac{1}{2}\left(e_{1}^{2} \nu_{1}^{2}+\cdots+e_{N} \nu_{N}^{2}\right)
$$


3) For $\varphi_{1}^{*}=\varphi_{10}$ and for small $\varepsilon$, the solution $\Omega_{1}=0, \varphi_{1}=\varphi_{10}$ is stable if

$$
k^{2} \cos \varphi_{10}+\frac{1}{2}\left(e_{1}^{2} \nu_{1}^{2}+\cdots+e_{N}^{2} \nu_{N}^{2}\right) \cos 2 \varphi_{10}>0 .
$$

Taking into account the relation (3.6):

$$
\cos \varphi_{10} \approx-\frac{2 k^{2}}{\left(e_{1}^{2} \nu_{1}^{2}+\cdots+e_{N}^{2} \nu_{N}^{2}\right)}
$$

one can write the stability condition in the form

$$
k^{2}>\frac{1}{2}\left(e_{1} \nu_{1}^{2}+\cdots+e_{N} \nu_{n}^{2}\right)
$$

which is in the contrary with (3.7).

Thus, with small $\varepsilon$ and for $k^{2} \approx \frac{1}{2}\left(e_{1}^{2} \nu_{1}^{2}+\cdots+e_{N}^{2} \nu_{N}^{2}\right)$ the stability conditions (3.9), (3.10) are similar to those in the non-resonance case.

\section{§4. CONCLUSION}

1. The downward position of equilibrium $(\Omega=0, \varphi=0)$ of the pendulum is always stable. The midle equilibrium position $\left(\Omega=0, \varphi=\varphi_{0}\right)$ of the pendulum is unstable. The upward equilibrium position $(\Omega=0, \varphi=\pi)$ of the pendulum is stable if there exists the condition (2.9) or (3.10).

2. The presence of vertically moving components of the support point intensifies the stability of downward and upward positions of equilibrium of the pendulum (see (3.9), (3.10)).

This paper was supported in part by the National basic research program in natural sciences.

\section{REFERENCE}

1. Nguyen Van Dinh. The stability of the inverse pendulum. Journal of Mechanics, No 4, 1993.

2. Bogoliubov N. N., Mitropolskii Yu. A. Asymptotic methods in the theory of nonlinear oscillations, Moscow, 1964.

Received November 25, 1993

\section{DAO ĐỘNG PHI TUYẾN CƯA CON LẮC CÓ ĐIỂM TREO DICH CHUYỂN THĂNG ĐỨNG ĐA TẦN}

Trong bài báo, các kết quả của [1] được mở rộng cho trường hợp điểm treo của con lắc thực hiện dao động thẳng đứng đa tần. Để giải quyết trọn vẹn bài toán về sự ổn định của các vị trí cân bằng của con lắc đã phải xét đến xấp xỉ cấp cao. Kết quả nghiên cứu cho thấy:

1. Vị trí cần bằng phía dưới của con lắc luôn luôn ởn định. Vị trí cân bằng trung gian không ổn định. Vị trí cân bằng phía trên ổn định nểu điều kiện (3.10) được thỏa mãn.

2. Sự xuất hiện các thành phần dao động đa tần của điểm treo cưa con lắc đã làm tăng tính ổn định của các vị trí cân bằng phía dưới và phía trên (xem (3.9), (3.10)). 